\title{
An Analysis of the Impact of Information and Communication Technologies on Non- Maintenance Shopping Activities
}

\author{
Chandra R. Bhat \\ The University of Texas at Austin, Dept of Civil Engineering \\ 1 University Station C1761, Austin, Texas, 78712-0278
}

Tel: 512-471-4535, Fax: 512-475-8744, Email: bhat@mail.utexas.edu

Aruna Sivakumar

The University of Texas at Austin, Dept of Civil Engineering

1 University Station C1761, Austin, Texas, 78712-0278

Tel: 512-471-4535, Fax: 512-475-8744, Email: arunas@mail.utexas.edu

\author{
Kay W. Axhausen \\ Verkehrsplanung (IVT), HIL F 32.3 \\ ETH Hönggerberg, CH - 8093 Zürich
}

Tel: +41-1-633 3943, Fax: +41-1-633 1057, Email: axhausen@ivt.baug.ethz.ch 


\title{
An Analysis of the Impact of Information and Communication Technologies on Non- Maintenance Shopping Activities
}

\author{
Chandra R. Bhat, Aruna Sivakumar, and Kay W. Axhausen
}

\begin{abstract}
This paper examines the use and travel impacts of two forms of Information and Communication Technologies (ICTs): mobile telephones and computers. The travel impacts are examined in the context of participation in out-of-home non-maintenance shopping activities over a multiweek period through the modeling of the duration between successive shopping activity participations. The empirical analysis uses a continuous six-week travel survey collected in the cities of Halle and Karlsruhe in Germany in the Fall of 1999. The results indicate that the effects of ICTs on activity-travel patterns are mediated by individual sociodemographic and locational factors, as well as by unobserved individual characteristics. The results also show that the substitution between mobile phone use and shopping travel is grossly underestimated if the effects of common unobserved factors affecting mobile phone use and shopping travel are not considered. In addition, there is quite substantial intra-individual variation in intershopping duration.
\end{abstract}

Keywords: Information and communication technologies, sample selection, random-coefficients, unobserved heterogeneity, hazard-based duration model, multiday analysis, activity-travel behavior, shopping behavior. 


\section{INTRODUCTION}

The rapid innovations and advances in information and communication technologies (ICTs) since the early 1990s have had a profound impact on the behavior of businesses and individuals in today's society. Businesses are increasingly harnessing the potential of ICTs to facilitate and consummate business-to-business transactions and business-to-consumer transactions. In particular, it is fairly routine today for businesses to manage the production and distribution activities of their services and products (supply-chain management), as well as promote the purchase of their services and products (electronic enabled commerce), through the use of the internet. Individuals and households are also substantially more likely today than ten years ago to use computers at home with web access and/or to use mobile telephony services, due in large part to the increasing affordability and functionality of these technologies. Projections suggest that technology improvements will further fuel the adoption and use of internet computing and mobile communication devices by individuals and households in the future. For example, broadband internet connections (cable modems and digital subscriber lines) to homes in the U.S. facilitate faster access to information, and the number of such connections is projected to increase by about thirtyfold between 2000 and 2007 (Pioneer Consulting, 1999). Similarly, the penetration of mobile telephones in the U.S. population is projected to increase from about $25 \%$ today to about 55\% in 2004 (Forrester, 2000).

The impacts of ICT adoption and use are likely to be far-reaching, with the potential to fundamentally alter the life styles of businesses and individuals (see, for example, Droege, 1997, Graham and Marvin, 1996, Boden, 1999, French et al., 1999 and Zimmerman et al., 2001, for broad discussions of the potential influence of ICTs on urban planning, medical care and services, education, and family responsibilities). An interesting aspect of ICT use from a 
transportation perspective is its impact on personal activity-travel behavior. Mokhtarian and Salomon (2002) develop a typology for studies in the area of ICT use and personal activity-travel behavior interrelationships. From a time horizon standpoint, she identifies the impact of ICT use on individual activity-travel behavior as being short-term direct effects or long term indirect effects through changes in work arrangements, residential locations choices, and car ownership levels. Most empirical studies to date have focused on the short-term direct effects, though there have been a few studies directed toward the long term indirect effects on activity-travel behavior (see Lund and Mokhtarian, 1994; Salomon, 1996; Graham and Marvin, 1996). In terms of breadth of analysis, Mokhtarian classifies earlier studies into comprehensive studies that attempt to uncover the interrelationships between ICT use and overall activity-travel behavior of individuals, or limited studies that focus on a small subset of choices characterizing ICT use and/or activity-travel impacts of ICT use. Almost all earlier empirical studies may be classified under the category of limited analysis studies, though some relatively comprehensive studies have been undertaken recently (Harvey and Taylor, 2000 and Mokhtarian and Meenakshisundaram, 1999). Finally, in the context of the scale of analysis, Mokhtarian classifies studies as macro-scale (regional, national, or international levels of analysis) or micro-scale (individual-level). Both scales of analysis have been used quite widely in the past.

In this study, we focus on the short-term direct interrelationship between ICT use and individual activity-travel behavior. Our study is also limited to the use of two kinds of ICT; mobile telephony and personal computer use; and to out-of-home shopping activities of individuals. We use a micro-scale level in the analysis. 
In the next section, we position the current study in the context of earlier studies from a substantive perspective. In Section 1.2, we highlight the methodological characteristics of our modeling framework.

\subsection{The Current Research in the Context of Earlier Research}

There have been several earlier studies of ICT use and the impact of ICT use on personal activity-travel behavior. These studies have provided important and useful insights into the interrelationships between ICT use and individual activity-travel patterns. The focus of this paper is to contribute to this growing body of ICT-related research. In addition, the current study may be distinguished from previous short-term, limited analysis, micro-scale ICT-related studies in several ways. First, the study focuses on an analysis of ICT use as well as the generation of outof-home shopping activities in a joint framework. Most earlier studies, on the other hand, have either focused only on ICT use or only on the impact of ICTs on activity-travel behavior ${ }^{1}$. These earlier studies do not account for the possible endogeneity of ICT use decisions in analyzing activity-travel patterns and, therefore, may not capture the true impact of ICT use on activitytravel patterns. For example, it is possible that an individual who has an active lifestyle may be more likely to use mobile phones, as well as be more likely to participate in (out-of-home) shopping activities. If ICT use is considered exogenous to the analysis of out-of-home shopping participation, one might find a "spurious" dependence of ICT use on shopping activity generation due to the active lifestyle characteristic. This, of course, can imply inappropriate

\footnotetext{
${ }^{1}$ Examples of studies of ICT use include Yen and Mahmassani (1998), Mokhtarian and Salomon (1997) and Mannering and Mokhtarian (1995) in the context of telecommuting, and Koppelman et al. (1991) and Manski and Salomon (1987) in the context of teleshopping/other ICT use. Examples of the impact of ICTs on activity-travel behavior include Henderson and Mokhtarian (1996), Pendyala et al. (1991), Koenig et al. (1996) and Mokhtarian and Varma (1998) in the context of the impact of telecommuting on commute-related activity-travel patterns, and Viswanathan and Goulias (2001), Tacken (1990), Gould and Golob (1997), Kilpala et al. (2000), Martens and Korver (2000), Yim (2000), and Handy and Yantis (1997) in the context of the impact of computing, internet access, and mobile technology on non-work activity-travel behavior.
} 
conclusions about the substitution or complementary effects of ICT use on shopping activity generation. Second, the current study allows the effect of ICT use to vary across individuals. Specifically, it allows the effect of ICT use to be in opposite directions for different individuals, so that ICT use substitutes for shopping trips for some individuals and complements shopping trips for others. Further, within the group of individuals for whom there is a substitution effect (or for whom there is a complementary effect), the current study accommodates heterogeneity in responsiveness to ICT use (the reader is referred to Chamberlain, 1980, Hsiao, 1986 and Diggle et al., 1994, for a detailed discussion of heterogeneity-related issues in statistical models that are non-linear in the model parameters). Third, the current study uses a multi-week activity-travel diary collected in Germany in the Fall of 1999 to evaluate the effects of ICT use on out-of-home shopping activity episodes. The study is, therefore, able to accommodate intra-individual variations in activity travel patterns over a multiday period. The analysis of such intra-individual variation is important to examine the frequency of exposure of different sociodemographic and travel segments to policy scenarios (see Jones and Clark, 1988). Fourth, the current study focuses on examining the impacts of two different kinds of interactive ICT activities (computer use and mobile phone use) on non-maintenance shopping trips (i.e., trips not associated with grocery shopping, medical drug shopping, etc.). Previous studies have mainly focused on the effect of a single ICT, generally associated with work-related computer use. ${ }^{2}$ Further, as noted by Handy and Yantis (1997), the impact of ICTs on nonwork travel "has been largely ignored by researchers so far". The one exception is the area of teleshopping; however, much of this teleshopping literature has concentrated on the interaction between online grocery shopping and

\footnotetext{
${ }^{2}$ Two recent studies have considered the impact of multiple ICTs, including mobile telephony (see Mokhtarian and Meenakshisundaram, 1999 and Viswanathan and Goulias, 2001); however, these studies do not distinguish between activity purposes. The current study focuses specifically on the interrelationship between non-maintenance shopping activities and ICT-use.
} 
out-of-home grocery shopping (see Golob, 2000). There has been little to no empirical research on the effect of ICTs on non-maintenance shopping behavior. Fifth, the current study examines shopping activity participation behavior by analyzing the duration between successive out-ofhome shopping activity participations of individuals. The intershopping duration is measured in days, since almost all individuals have no more than a single shopping activity participation on any given day. The duration-based structure used in this paper recognizes the dynamics of intershopping duration; that is, it recognizes that the likelihood of participating in shopping activity depends on the length of elapsed time since the previous participation. The traditional "number of trips" formulation is unable to accommodate such dynamics. The duration formulation also allows different individuals to have different participation rhythms in shopping behavior and it can be used to predict the effects of ICT on shopping activity participation behavior over any period of time (such as a day, a week, or a month).

An important point to note here is that our representation of ICT use is constrained by the ICT-related data collected in the six-week German diary data. Specifically, mobile telephone use is represented by the availability (i.e., adoption) of a mobile telephone. Similarly, home computer use is broadly represented by whether the individual has access to personal e-mail at home. These representations of ICT use are not as rigorous as those of some earlier studies that use detailed diary data on the number and attributes of communication activities by ICT type (see Moberg, 1993; Spittje, 1994; Zumkeller, 1996; Mokhtarian and Meenakshisundaram, 1999).

\subsection{Methodological Characteristics of Modeling Framework}

The current paper uses a hazard-based model to examine the effect of ICTs on intershopping duration, while controlling for sociodemographic attributes of individuals and their residential 
location attributes (see Hensher and Mannering, 1994 and Bhat, 2000 for an extensive discussion of hazard-based duration models and transportation-related applications). The hazard formulation developed and applied in this paper extends the hazard formulations of Han and Hausman (1993) and Bhat (1996) in several ways. First, the paper accommodates sample selection in duration based on multiple criteria (in the application in the paper, the multiple criteria for sample selection are associated with the adoption of multiple ICTs). Second, the paper recognizes that the criteria for sample selection are individual-specific, but that the resulting sample selection affects all intershopping durations of the individual. This is an important difference from usual "cross-sectional" sample selection models in which both the criteria and variable of interest are associated with the same unit of observation (for example, see Greene, 2000; Pages 926-936). Third, the paper accommodates heterogeneity in sensitivity to ICTs due to observed as well as unobserved individual attributes. Fourth, the model in the paper allows unobserved heterogeneity across individuals and across spells of the same individual in intershopping duration. To our knowledge, this is the first formulation and application of a duration model in econometric literature to consider the above issues in a comprehensive, unifying, framework. Fifth, the estimation of the duration model requires simulation techniques to evaluate the multidimensional integral in the estimation criterion function. In the current paper, we use a simulation technique based on "intelligent" quasi-random draws rather than "random draws".

The remainder of this paper is structured as follows. The next section discusses the model structure and estimation technique. Section 3 presents the data source and describes the sample used in the analysis. Section 4 discusses the empirical results. The final conclusion section summarizes the important findings from the study and identifies directions for further research. 


\section{MODEL STRUCTURE}

In the following presentation, we will first discuss the hazard-based model structure for intershopping duration without considering the sample selection based on ICT use. We will then introduce the modeling of ICTs along with the specification for the sample selection of intershopping duration based on ICT use.

\subsection{Hazard-Based Duration Structure}

Let $T_{q i}$ represent the continuous duration time of the $i^{\text {th }}$ intershopping spell of individual $q$. Let $\tau$ represent some specified time on the continuous time scale. Let $\lambda_{q i}(\tau)$ represent the hazard at continuous time $\tau$ since the previous shopping activity participation for the $i^{\text {th }}$ intershopping duration spell of individual $q$; i.e., $\lambda_{q i}(\tau)$ is the conditional probability that individual $q$ 's $(i+$ $1)^{\text {th }}$ shopping episode will occur at continuous time $\tau$ after her/his $i^{\text {th }}$ participation, given that the episode does not occur before time $\tau$ :

$\lambda_{q i}(\tau)=\lim _{\Delta \rightarrow 0^{+}} \frac{P\left(\tau<T_{q i}<\tau+\Delta \mid T_{q i}>\tau\right)}{\Delta}$

Next, we relate the hazard rate, $\lambda_{q i}(\tau)$, to a baseline hazard rate, $\lambda_{0}(\tau)$, a scalar $\alpha_{q}$ capturing socio demographic and other attributes of individual $q$, a vector of ICT-use covariates, $x_{q}$, and a spell-specific unobserved component $\varpi_{q i}\left(\Phi_{q i}\right.$ corresponds to random noise across different intershopping durations). We accomplish this by using a proportional hazard formulation as follows:

$\lambda_{q i}(\tau)=\lambda_{0}(\tau) \exp \left(-\alpha_{q}-\beta_{q}^{\prime} x_{q}+\Phi_{q i}\right)$, 
where $\beta_{q}$ is a vector of individual specific ICT-use coefficients. For reasons that will become clear later, we assume a gamma distribution for $\exp \left(\varpi_{q i}\right)$. The exponential specification in Equation (2) guarantees the positivity of the hazard function without placing constraints on the sign of $\alpha_{q}$ and the elements of the vector $\beta_{q}$.

The proportional hazard formulation of Equation (2) can be written in the following equivalent form (see Bhat, 2000):

$$
s_{q i}^{*}=\ln \int_{\tau=0}^{T_{q i}} \lambda_{0}(\tau) d \tau=\alpha_{q}+\beta_{q}^{\prime} x_{q}-\bar{\varpi}_{q i}+\varepsilon_{q i}
$$

where $\varepsilon_{q i}$ is a random term with an extreme value distribution: $\operatorname{Prob}\left(\varepsilon_{q i}<z\right)=F_{\varepsilon}(z)=1-\exp [-$ $\exp (z)]$

Now, consider the case when the continuous variable $T_{q i}$ is unobserved in Equation (3). However, we do observe the discrete time intervals of intershopping duration, where the discrete interval is in the unit of a day. Let $t_{q i}$ represent discrete time intervals of intershopping duration, where the discrete interval is in the unit of a day. Let $t_{q i}$ represent the $i^{\text {th }}$ intershopping duration (in days) of individual $q$ and let $k$ be an index for days (thus, $t_{q i}=1,2, \ldots k, \ldots K$, where $k$ is in days). Defining $\tau^{k}$ as the continuous time representing the upper bound of the $k^{\text {th }}$ day, we can write

$$
s_{q i}^{*}=\ln \int_{\tau=0}^{T_{q i}} \lambda_{0}(\tau) d \tau=\alpha_{q}+\beta_{q}^{\prime} x_{q}-\bar{\omega}_{q i}+\varepsilon_{q i}, t_{q i}=k \text { if } \psi_{k-1}<s_{q i}^{*}<\psi_{k}, \psi_{k}=\ln \int_{\tau=0}^{\tau^{k}} \lambda_{0}(\tau) d \tau
$$

A number of different specifications may be used for the coefficient vectors $\alpha_{q}$ and $\beta_{q}$ in Equations (2) and (3). The simplest specification is $\alpha_{q}=0$ and $\beta_{q}=0$ for all individuals, and $\varpi_{q i}=0$ for all intershopping duration spells. This, of course, corresponds to the Kaplan-Meier 
sample hazard. A second specification is to write $\alpha_{q}$ as a deterministic function of an observed vector $w_{q}$ of individual sociodemographic and residential location attributes $\left(\alpha_{q}=\delta^{\prime} w_{q}\right)$, and to allow heterogeneity across individuals in the effect of ICT use on the intershopping hazard due to observed individual characteristics by specifying the ICT use coefficient $\beta_{q l}(l=1,2, \ldots, L)$ as a function of an observed vector $y_{q l}$ of individual attributes: $\beta_{q l}=\vartheta_{l}+\gamma_{l}^{\prime} y_{q l}$. The spell-specific error term $\Phi_{q i}$ is included in this formulation. The variance of $\Phi_{q i}$ captures the level of heterogeneity in intershopping hazard across all spells. We will refer to this specification as the deterministic coefficients duration (DCD) model. A third specification superimposes random (unobserved) individual heterogeneity over the deterministic (observed) heterogeneity of the DCD model: $\alpha_{q}=\delta^{\prime} w_{q}+v_{q}$ and $\beta_{q l}=\vartheta_{l}+\gamma_{l}^{\prime} y_{q l}+\eta_{q l}$, where $v_{q}$ and $\eta_{q l}$ are assumed to be normally distributed across individuals; $\left[v_{q} \sim N\left(0, \sigma_{v}^{2}\right) ; \eta_{q l} \sim N\left(0, \sigma_{\eta^{l}}^{2}\right)\right]$. In addition, we assume that $v_{q}$ is independent of each $\eta_{q l}$ random term $(l=1,2, \ldots, L)$ and that the $\eta_{q l}$ terms are independent of each other; $v_{q}$ and $\eta_{q l}$ represent individual-specific unobserved factors associated with overall preferences for shopping and sensitivity to ICT-use, respectively; the variance of $\Phi_{q i}$ in this third specification captures within-individual heterogeneity in the intershopping hazard. We will refer to the random specification above as the random coefficients duration $(\mathrm{RCD})$ model.

\subsection{Sample Selection with Hazard Based Duration Structure}

In the previous section, ICT use was considered exogenous to intershopping duration. However, as indicated earlier in Section 1.1, considering ICT use to be exogenous may lead to an incorrect assessment of the effect of ICT use on intershopping duration. This is because the intershopping 
hazard model of the previous section does not disentangle the true effect of ICT adoption from the spurious effect of ICT adoption. The latter effect corresponds to common unobserved factors that influence ICT adoption as well as the intershopping hazard. In this section, we discuss a sample selection model structure that considers the endogenous nature of ICT use.

For ease in presentation, we present the sample selection model structure considering only two ICT adoption variables; mobile phone use and computer use at home (these are also the two ICT adoption variables used in our empirical analysis). However, the framework is general and can be extended to multiple ICT-use variables without any conceptual or practical difficulty.

The equation system for the sample selection model structure comprises three equations: one equation each for the mobile phone and computer use choices, and a third equation for the intershopping duration modeling. Considering the form of the proportional hazard formulation in Equation (3) for intershopping duration, the equation system may be written as follows:

$$
\begin{aligned}
& m_{q}^{*}=\theta^{\prime} h_{q}+\zeta_{q}+v_{q}, m_{q}=1 \text { if } m_{q}^{*}>0, m_{q}=0 \text { if } m_{q}^{*} \leq 0 \\
& p_{q}^{*}=\mu^{\prime} r_{q}+\xi_{q}+\omega_{q}, p_{q}=1 \text { if } p_{q}^{*}>0, p_{q}=0 \text { if } p_{q}^{*} \leq 0 \\
& s_{q i}^{*}=\alpha_{q}+\beta_{q}^{\prime} x_{q} \pm \zeta_{q} \pm \xi_{q}-\varpi_{q i}+\varepsilon_{q i}, t_{q i}=k \text { if } \Psi_{k-1}<s_{q}^{*}<\psi_{k}, \Psi_{k}=\ln \int_{\tau=0}^{\tau^{k}} \lambda_{0}(\tau) d \tau, x_{q}=\left[m_{q}, p_{q}\right]^{\prime},
\end{aligned}
$$

where $m_{q}^{*}$ and $p_{q}^{*}$ are latent propensities to use mobile telephones and computers, respectively, and $m_{q}$ and $p_{q}$ are dummy variables representing whether or not an individual uses mobile phones and computers, respectively. $h_{q}$ and $r_{q}$ are column vectors of exogenous variables affecting mobile telephone use and computer use, and $\theta$ and $\mu$ are corresponding column vectors to be estimated. $v_{q}$ and $\omega_{q}$ are standard normal variables with a correlation $\rho$. This correlation term captures common unobserved factors that affect the propensity to use mobile 
telephones and a personal computer at home. $\zeta_{q}$ is a normal random error term that captures common unobserved factors influencing mobile phone use propensity and the intershopping hazard $\left[\zeta_{q} \sim N\left(0, \sigma_{\zeta}^{2}\right)\right]$. This term causes "spurious" dependence in mobile phone use and the intershopping hazard. The ' \pm ' sign in front of $\zeta_{q}$ in the hazard equation indicates that the correlation in unobserved factors between mobile phone use and intershopping hazard may be positive or negative. If the sign is ' + ', it implies that individuals who use mobile phones are also intrinsically more likely to have a lower intershopping hazard (see the correspondence between Equations 2 and 3). Equivalently, it implies that individuals who use mobile phones are intrinsically more likely to have longer intershopping durations, or fewer shopping trips. If the sign is '-', it implies that individuals who use mobile phones are intrinsically more likely to have a higher intershopping hazard, i.e., they are more likely to have shorter intershopping durations or undertake more shopping trips. Of course, if such correlations are ignored, they "corrupt" the "true" dependence of the intershopping hazard on mobile phone use. This issue is discussed in more detail in the empirical results section. $\xi_{q}$ is a normal random term that similarly captures common unobserved factors influencing personal computer use propensity and the intershopping hazard; $\xi_{q} \sim N\left(0, \sigma_{\xi}^{2}\right)$

\subsection{Model Estimation}

The parameters to be estimated in the sample selection model with a random coefficient duration (RCD) structure include the $\theta$ and $\mu$ vectors, the $\delta, \vartheta$, and $\gamma$ vectors in the duration model, the $\psi$ thresholds in the duration model that provide information regarding the baseline intershopping hazard profile, the $\rho$ correlation parameter capturing the effect of common 
unobserved factors that affect the propensity to use mobile telephones and computers at home, the scalar variance terms $\sigma_{v}^{2}, \sigma_{\xi}^{2}, \sigma_{\xi}^{2}$, and the vector variance term $\sigma_{\eta}^{2}$. Let $\Omega$ represent a vector that includes all these parameters to be estimated, and let $\Omega_{-\sigma}$ represent a vector of all parameters except the variance terms. Define $g_{q}=2 m_{q}-1$ and $n_{q}=2 p_{q}-1$. Then the likelihood function for a given value of $\Omega_{-\sigma}$ and the error terms $\zeta_{q}, \xi_{q}, \eta_{q}, \nu_{q}$, and $\varpi_{q i}$ may be written for an individual $q$ 's $i^{\text {th }}$ intershopping duration spell as:

$$
\begin{aligned}
L_{q i}\left(\Omega_{-\sigma}\right) \mid \zeta_{q}, \xi_{q}, \eta_{q}, v_{q}, \varpi_{q i}= & \Phi_{2}\left[g_{q} \cdot\left(\theta^{\prime} h_{q}+\zeta_{q}\right), n_{q} \cdot\left(\mu^{\prime} r_{q}+\xi_{q}\right), g_{q} n_{q} \rho\right] \times \\
& {\left[\left(\exp \left\{-B_{\left(t_{q i}-1\right)} \exp \left(\varpi_{q i}\right)\right\}\right)-\left(\exp \left\{-B_{t_{q i}} \exp \left(\varpi_{q i}\right)\right\}\right)\right], }
\end{aligned}
$$

where $t_{q i}$ is the actual intershopping duration of individual $q$ in the $i^{\text {th }}$ spell, $\Phi_{2}($.$) is the$ bivariate cumulative standard normal distribution, and

$$
B_{t_{q i}}=\exp \left\{\psi_{t_{q i}}-\left[\delta^{\prime} w_{q}+\sum_{l}\left(\vartheta_{l}+\gamma_{l}^{\prime} y_{q l}+\eta_{q l}\right) x_{q l} \pm \zeta_{q} \pm \xi_{q}+v_{q}\right]\right\}
$$

Assuming that $c_{q i}\left[=\exp \left(\varpi_{q i}\right)\right]$ is distributed as a gamma random variable with a mean one (a normalization) and variance $\sigma_{c}$, the likelihood function for individual $q$ 's $i^{\text {th }}$ intershopping duration spell, unconditional on $\varpi_{q i}$, may be written as:

$$
\begin{aligned}
L_{q i}\left(\Omega_{-\sigma}\right) \mid \zeta_{q}, \xi_{q}, \eta_{q}, v_{q}= & \Phi_{2}\left[g_{q} \cdot\left(\theta^{\prime} h_{q}+\zeta_{q}\right), n_{q} \cdot\left(\mu^{\prime} r_{q}+\xi_{q}\right), g_{q} n_{q} \rho\right] . \\
& \left\{\int_{0}^{\infty}\left[\exp \left\{-B_{t_{q i-1}} \cdot c_{q i}\right\}-\exp \left\{-B_{t_{q i}} \cdot c_{q i}\right\}\right] f\left(c_{q i}\right) d c_{q i}\right\}
\end{aligned}
$$

Using the moment-generating function properties of the gamma distribution (see Johnson and Kotz, 1970), the expression above reduces to:

$$
L_{q i}\left(\Omega_{-\sigma}\right) \mid \zeta_{q}, \xi_{q}, \eta_{q}, \nu_{q}=\Phi_{2}\left[g_{q} \cdot\left(\theta^{\prime} h_{q}+\zeta_{q}\right), n_{q} \cdot\left(\mu^{\prime} r_{q}+\xi_{q}\right), g_{q} n_{q} \rho\right] \cdot\left[G_{\left(t_{q i}-1\right)}-G_{t_{q i}}\right],
$$

where $G_{t_{q i}}=\left[1+\sigma_{c}^{2} B_{t_{q i}}\right]^{-\sigma^{-2}}$. The gamma distribution for $c_{q i}$ is convenient because it results in a closed-form expression in Equation (8). Next, define the following standard normal variables: 
$f_{q v}=\frac{v_{q}}{\sigma_{v}}, f_{q \zeta}=\frac{\zeta_{q}}{\sigma_{\zeta}}, f_{q \xi}=\frac{\xi_{q}}{\sigma_{\xi}}$, and $f_{q q l}=\frac{\eta_{q l}}{\sigma_{\eta l}}(l=1,2$; the range of $l$ corresponds to the number of ICTs). Also, define $f_{q \mathrm{~m}}=\left(f_{q \eta 1}, f_{q \mathrm{q} 2}\right)^{\prime}$. Then the likelihood function for a given value of the parameter vector $\Omega$ and for an individual $q$ with $I_{q}$ intershopping duration spells can be written conditional on $f_{q 5}, f_{q 5}, f_{q v}$, and the $f_{q \eta}$ random terms as:

$L_{q}(\Omega) \mid f_{q \zeta}, f_{q \xi}, f_{q \mathrm{v}}, f_{q \eta}=\prod_{i=1}^{I_{q}}\left\{\Phi_{2}\left[g_{q} \cdot\left(\theta^{\prime} h_{q}+f_{q \zeta} \sigma_{\zeta}\right), n_{q} \cdot\left(\mu^{\prime} r_{q}+f_{q \xi} \sigma_{\xi}\right), g_{q} n_{q} \rho\right] \cdot\left[G_{\left(t_{q i}-1\right)}-G_{t_{q i}}\right]\right\}$,

where $G_{t_{q i}}=\left[1+\sigma_{c}^{2} B_{t_{q i}}\right]^{-\sigma^{-2}}$

and $B_{t_{q i}}=\exp \left\{\psi_{t_{q i}}-\left[\delta^{\prime} w_{q}+\sum_{l}\left(\rho_{l}+\gamma_{l}^{\prime} y_{q l}+f_{q \eta l} \sigma_{\eta l}\right) x_{q l} \pm f_{q \zeta} \sigma_{\zeta} \pm f_{q \xi} \sigma_{\xi}+f_{q v} \sigma_{v}\right]\right\}$

The unconditional likelihood for individual $q$ with $I_{q}$ intershopping durations may finally be written as:

$L_{q}(\Omega)=\int_{f_{q \zeta}-\infty f_{q}}^{+\infty} \int_{q \zeta-\infty}^{\infty} \int_{q q}^{\infty} \int_{-\infty}^{\infty} \int_{q \eta}^{\infty}\left[L_{q}(\Omega) \mid f_{q \xi}, f_{q \xi}, f_{q v}, f_{q \eta}\right] \cdot d \Phi\left(f_{q \xi}\right) d \Phi\left(f_{q \xi}\right) d \Phi\left(f_{q v}\right) d \Phi\left(f_{q \eta}\right)$.

The log-likelihood function is $\mathscr{L}(\Omega)=\sum_{q} \ln L_{q}(\Omega)$.

The likelihood function in Equation (10) involves the evaluation of an ( $L+3)$-dimensional integral, where $L$ is the number of ICTs modeled in the analysis. In the current empirical analysis, there are two ICTs, leading to a five-dimensional integral. This five-dimensional integration cannot be accomplished using general purpose numerical methods such as quadrature, since quadrature techniques cannot evaluate the integrals with sufficient precision and speed for estimation via maximum likelihood (see Hajivassiliou and Ruud, 1994).

We apply simulation techniques to approximate the integrals in the individual-specific likelihood function and maximize the logarithm of the resulting individual-specific simulated 
likelihood function across individuals with respect to $\Omega$. The simulation technique approximates the individual likelihood function in Equation (10) by computing the integrand in the equation at different realizations of $f_{q \zeta}, f_{q \xi}, f_{q v}$, and $f_{q \eta_{l}}$ drawn from standard normal distributions, and computing the individual likelihood function by averaging over the different values of the integrand across the different realizations. Notationally, if $S L_{q}^{d}(\Sigma)$ is the realization of the individual likelihood function in the $d$ th draw $(d=1,2, \ldots, D)$, then the individual likelihood function is approximated as:

$S L_{q}(\Omega)=\frac{1}{D} \sum_{d=1}^{D} S L_{q}^{d}(\Omega)$,

where $S L_{q}(\Omega)$ is the simulated likelihood function for the $q$ th individual's sequence of intershopping episodes, given the parameter vector $\Omega . S L_{q}(\Omega)$ is an unbiased estimate of the actual likelihood function $L_{q}(\Omega)$. Its variance decreases as $D$ increases. It also has the appealing properties of being smooth (i.e., twice differentiable) and being strictly positive for any realization of the draws.

The simulated log-likelihood function is constructed as:

$S \mathscr{L}(\Omega)=\sum_{q} \ln \left[S L_{q}(\Omega)\right]$.

The parameter vector $\Omega$ is estimated as the value that maximizes the above simulated function. Under rather weak regularity conditions, the maximum (log) simulated likelihood (MSL) estimator is consistent, asymptotically efficient, and asymptotically normal (see Hajivassiliou and Ruud, 1994; Lee, 1992).

In the current paper, we use a quasi-Monte Carlo (QMC) method to draw realizations for $f_{q \zeta}, f_{q \xi}, f_{q v}$, and $f_{q \eta_{l}}$ from their population normal distributions. The QMC approach uses 
"cleverly" crafted non-random and more uniformly distributed sequences within the domain of integration. The underlying idea of the method is that it is really inconsequential whether the discrete points are truly random; of primary importance is the even distribution (or maximum spread) of the points in the integration space. The sequences used in the quasi-Monte Carlo (or QMC) method are labeled as quasi-random sequences, though this is a misnomer since randomness plays no part in the construction of the sequences. Within the broad framework of QMC sequences, we specifically use 125 draws of the Halton sequence in the current analysis. Details of the Halton sequence and the procedure to generate this sequence are available in Bhat, (2001). Bhat has demonstrated that the Halton simulation method out-performs the traditional pseudo-Monte Carlo (PMC) methods for mixed logit model estimation. Subsequent studies by Train (1999) and Hensher (1999) have confirmed this result.

\section{THE DATA}

\subsection{Data Source}

The data source for the current study is a 6-week travel survey conducted in Karlsruhe (West Germany) and Halle (East Germany) as part of the MobiDrive study funded by the German Ministry for Research and Education (see Axhausen et al., 2002, for a detailed description of this data source). The main objective of this travel survey data collection was to facilitate a better understanding of the rhythms, routines, and habits of individuals over an extended time period of several weeks. The data collection effort was initiated by contacting a sample of households randomly selected from a phonebook database in each of the two cities. A subsample of this larger sample of households was selected for administration of the travel survey, based on eligibility considerations and willingness to participate (only households who did not plan to take 
a vacation of more than a week during the survey period and who did not have children under the age of 6 years were deemed eligible).

The final sample from the survey included information on 361 individuals from 162 households. Of these, 44 individuals from 23 households in Karlsruhe participated in a pretest survey, and 317 individuals from 139 households in Karlsruhe and Halle participated in the main survey. The structure and administration procedures were identical in the two surveys. The pretest travel survey was administered between May 31st and July 25th, and the main survey was administered between September 13th and November 14th. In addition to the six-week continuous travel diary, information on the sociodemographic characteristics of households and their members, car fleet size and composition, and attitudes toward different modes of transport was also collected (the reader is referred to Schlich et al. (2000) for a description of detailed information collected in the survey).

\subsection{Sample Used and Description}

The sample used in the current analysis comprises 1144 intershopping duration spells of 255 adult individuals (an adult individual is defined as one whose age is equal to or over 16 years; we restricted the empirical analysis to adult individuals on the basis that children are likely to be accompanied by adults for shopping activities, and are not likely to be decision-makers themselves). The intershopping spells correspond to participation in non-maintenance shopping activities (i.e., not including grocery shopping, medical drug shopping, etc.).

The number of intershopping duration spells over the course of the survey varies between 1 and 18 across individuals, with an average of 4.74 spells. The length of the intershopping duration varies between 1 and 40 days. However, there were very few intershopping spells larger 
than 23 days, and so we collapsed all these intershopping durations to 23 days (only 14 intershopping spells out of 1144 , or $1.2 \%$ of spells, were longer than 23 days).

The sample hazard function plotted in Figure 1 provides descriptive information on intershopping duration. The sample hazard associated with each period is computed, using the Kaplan-Meier (KM) nonparametric estimator, as the number of terminated episodes in period $t$ divided by the risk set in period $t$ (see Kiefer, 1988). The hazard reveals spikes at 7, 18, and 21 days, indicating a certain level of rhythmic interpurchase durations among shoppers. However, the overall hazard profile is quite flat, suggesting a substantial amount of randomness in interpurchase durations when the effect of covariates and unobserved heterogeneity are ignored (see discussion under Equation (3)).

\subsection{Variable Specification and Model Formulation}

Three broad sets of variables were considered in the analysis: individual characteristics, household characteristics, and location characteristics. Table 1 provides the definitions and sample statistics for the variables used in the final model specifications. The final model specifications were obtained based on a systematic process of eliminating statistically insignificant variables, parsimony in the representation of the effects of variables, and intuitive consideration.

In this paper, we present the results for two model formulations that correspond to: (1) Exogenous models for ICT use with a random coefficient specification for the intershopping hazard and (2) Endogenous models for ICT use with a random coefficient specification for the intershopping hazard, and with '-' signs on the $\zeta_{q}$ and $\xi_{q}$ terms in the hazard structure of Equation (4). We adopt a negative sign on the error $\zeta_{q}$ and $\xi_{q}$ terms because this combination 
provided a better data fit than the other three sign combinations on $\zeta_{q}$ and $\xi_{q}(i . e .$, the $[+,+],[+$, $-]$, and $[-,+]$ combinations). The '-' signs imply that individuals who use mobile phones and/or computers at home are intrinsically more likely to have a higher intershopping hazard; that is, individuals who use mobile phones and/or computers at home are intrinsically more likely to undertake shopping activities than individuals who do not use ICTs. As we will indicate empirically later, ignoring this correlation in ICT use and intershopping duration will, in general, lead to incorrect estimates of the impact of ICT use on intershopping duration.

\section{EMPIRICAL RESULTS}

The results are presented in four broad sections. The first section presents the results for the ICT use models. The second section discusses the results of the intershopping duration hazard model, and presents the sample selection and unobserved heterogeneity parameters. The reader will note that the estimation of the ICT use models, the duration hazard, and the sample selection and unobserved heterogeneity parameters are all achieved simultaneously. They are discussed in separate sections for presentation ease. The final section discusses model fit statistics.

\subsection{ICT Use Estimation Results}

Table 2 provides the estimation results for mobile phone use and home computer use. As can be observed, the coefficient estimates do not vary substantially among the two model structures (i.e., the exogenous ICT use-random coefficients hazard model and the endogenous ICT userandom coefficients hazard model). This is to be expected since the ICT use coefficients are consistently estimated regardless of whether or not sample selection of the duration model, based 
on the ICT use variables, is accommodated. The next two subsections discuss the results for mobile phone use and home computer use, respectively, in greater detail.

\subsubsection{Mobile phone use results}

The impact of individual characteristics on mobile phone use propensity in Table 2 indicates that employed individuals are more likely to use mobile phones relative to unemployed individuals. Similarly, individuals with high education are more likely to use mobile phones relative to individuals with low education. The impact of age is interesting. The results indicate that the highest level of mobile phone use is among teenage adults (16-19 years of age). Among individuals above 19 years, middle-aged individuals (30-50 years) are the most likely to use mobile phones. The lower level of mobile phone use in the age group of 20-29 compared to teenagers and middle-aged individuals is a little surprising, and deserves further exploration that is beyond the scope of the current study.

The effects of household attributes indicate that individuals in high income households use mobile phones more than individuals in low income households, as expected. In addition, individuals in households with several adults tend to be less likely to use mobile phones, perhaps because reaching an adult in such households in the case of emergencies is easier using conventional telephones than reaching an adult in households with few adults. The flexibility and convenience offered by mobile telephones would also facilitate the more efficient realization of family and personal responsibilities, which would be particularly important for households with fewer adults. Finally, among household characteristics, nuclear family households are much more likely to use mobile phones compared to other types of households. 
The results of locational characteristics show that households in Karlsruhe (in West Germany) are more likely to own mobile phones than those in Halle (in East Germany). This is perhaps a reflection of the better economic conditions in Karlsruhe compared to Halle. Further, individuals in households in urban-suburban and suburban locations are more likely to use mobile phones than individuals in urban and rural areas.

\subsubsection{Computer use at home}

The pattern of the impacts of individual, household, and locational attributes on computer use at home are similar to the impact of these variables on mobile phone use. The one additional variable is the single-person household variable. The effect of this variable suggests that individuals living alone are not as likely to use computers at home as individuals in non-single households.

\subsubsection{Correlation in use of ICTs}

The correlation in unobserved factors affecting mobile phone propensity and computer use propensity is positive and statistically significant (see the last row of Table 2); that is, the same unobserved factors that make an individuals inclined to use one type of ICT also make the individual inclined to use the other type of ICT. This is an intuitive result.

\subsection{Hazard-Based Duration Model Estimation Results}

\subsubsection{The baseline hazard}

The baseline hazard functions for the two different model formulations are provided in Figure 1. The overall profiles of these hazard functions are similar with spikes at 7, 18, and 21 days. Also, 
there is a heightened hazard profile between 12-16 days, i.e., around the two week intershopping duration.

There is one clear difference between the sample and baseline hazard functions in Figure 1. Specifically, the baseline hazard functions show an increasing trend as the intershopping duration increases, reflecting a "snowballing" effect due to depletion of inventory; that is, individuals are more likely to engage in shopping as the time elapsed since the previous participation increases.

\subsubsection{Effects of individual and household characteristics on intershopping hazard}

The effects of covariates on the intershopping hazard are provided in Table 3 . It should be observed from Equation (2) that a positive coefficient on a covariate implies that the covariate lowers the hazard rate, or equivalently, increases the intershopping duration. Alternatively, a negative coefficient on a covariate implies that the covariate increases the hazard rate, or equivalently, the covariate decreases the intershopping duration.

The effect of individual characteristics in Table 3 indicates that men have a lower hazard (i.e., a higher intershopping duration) than women, and shoppers who use a car as the primary mode to participate in shopping have a lower hazard than those who use other modes. The latter effect may reflect the ability to carry large amounts of shopping items if a car is used, resulting in less need to shop frequently. The results also indicate that individuals who chain shopping participations with other activity stops are more likely to shop frequently.

The effect of household characteristics indicates that individuals in high income households have a higher intershopping hazard (i.e., shop more frequently) than individuals in low income households. This is intuitive, and reflects the higher shopping expenditure potential 
of high income households. Household structure also affects the intershopping hazard, with individuals in couple family households likely to have a higher intershopping hazard compared to nuclear family and other households. This may be attributed to the lower household responsibilities in couple families, and the consequent higher mobility levels of individuals in such households.

The impact of individual and household characteristics on the intershopping hazard are similar across the different models. However, the impacts of the ICT use variables are quite different in the two models, as we discuss next.

\subsubsection{Effect of ICT use variables}

The effect of ICT use on the duration hazard, as estimated in each of the two models, is discussed in the subsequent two subsections. Within each subsection, the focus is on three issues: model coefficients, the percentage of individuals for whom ICT use has a substitution effect versus a complementary effect, and the mean percentage change in the hazard because of ICT use.

\subsubsection{Exogenous ICT use-random coefficient model}

Our initial explorations indicated virtually no variation in the effect of mobile phone use on the intershopping hazard due to unobserved individual factors and hence we do not include a random heterogeneity term for the effect of mobile phone use. However, the magnitude of the standard deviation characterizing the distribution of individual-specific effects of computer use on the intershopping hazard is sizeable compared to the mean computer use effect, as shown in Table 3. 
This estimated standard deviation parameter is also significant at about the $7 \%$ level of significance (see the standard deviation under "computer use" in the Table).

The parameters capturing the effect of mobile phone use in the exogenous ICT usedeterministic duration hazard model indicates that mobile phone use decreases the intershopping hazard (i.e., increases the intershopping duration or decreases the number of shopping trips) for individuals with low education levels and living in Halle (see the coefficient on the constant under mobile phone use $=0.3898$ ). Thus, mobile phone use has a substitution effect on shopping trips for such individuals. This substitution effect extends to individuals with high education and living in Halle $(0.3898-0.2714=0.1184)$, and individuals with low education and living in Karlsruhe $(0.3898-0.3002=0.0896)$. However, the effect turns to one of complementarity for individuals with high education and living in Karlsruhe $(0.3898-0.3002-0.2714=-0.1818)$.

The mean coefficient and the standard deviation on the computer use variable suggests that computer use has an overall substitution effect on number of shopping episodes; however, computer use has a complementary effect for about $22 \%$ of computer users.

To summarize, the exogenous ICT use-random coefficients model indicates homogeneity in the shopping behavior of mobile phone users, after controlling for their education levels and residential location. However, among computer users, 78\% make fewer shopping trips than nonusers, while $22 \%$ make more shopping trips than non-users.

The mean effect of ICT use on the intershopping hazard may be computed as $\left\{\overline{\exp \left(\beta_{l}\right)}-1\right\} \times 100$, where $\overline{\exp \left(\beta_{l}\right)}$ is the mean value of the exponent of $\beta_{l}$. Now, since $\beta_{l}$ is normally distributed in the random coefficients specification, $\exp \left(\beta_{l}\right)$ is log-normally distributed with mean:

$\overline{\exp \left(\beta_{l}\right)}=\exp \left(\vartheta_{l}+\gamma_{l}^{\prime} y_{q l}\right) \cdot\left[\exp \left(\sigma_{\eta l}^{2}\right)\right]^{0.5}$ 
The resulting estimates of the mean impacts of ICT use are provided in the column under the exogenous ICT use-random coefficient model in Table 4.

\subsubsection{Endogenous ICT use-random coefficient model}

The estimated coefficient on the mobile phone use constant in the endogenous ICT use-random coefficients model is higher in magnitude than in the exogenous ICT use-random coefficients model, as can be observed in Table 3. This reflects a substantially higher substitution effect due to mobile phone use. This is an intuitive result, when viewed in combination with the estimated highly significant standard deviation $(=0.4174)$ of the common unobserved error component between the mobile phone use propensity and the duration hazard (see under sample selection and heterogeneity terms in Table 3). Specifically, as discussed earlier, the significant presence of unobserved factors common to the duration hazard and mobile phone use propensity implies that mobile phone users are intrinsically likely to be making more shopping trips than non-users. If this association is ignored, the intrinsic complementarity in mobile phone use and shopping trips (due to unobserved individual characteristics, such as say active and dynamic lifestyle) reduces the magnitude of the true substitution effect of mobile phone use on shopping trips. As a simple illustration of this effect, assume two groups of individuals with identical observed characteristics. The first group of individuals has unobserved characteristics that lead to mobile phone use and an overall desire for a higher number of shopping episodes than the second group of mobile phone non-users. If individuals in the first group were not using mobile phones, they would have made, say, 6 trips. Individuals in the second group, however, make only 4 trips. Note that this difference in trip-making propensity is because of unobserved life-style related factors. If these unobserved factors are ignored, the implicit assumption is that both the first and second 
groups make only 4 trips. Now, because of mobile phone use in the first group of mobile phone users, we might actually observe individuals in this group making 3 trips. Thus, the true reduction in trips because of mobile phone use is from 6 trips to 3 trips, an overall substitution effect of 50\%. However, if the unobserved factors that caused the first group to be more shopping-inclined were ignored, the (mis-)estimated reduction in trips because of mobile phone use would be from 4 to 3 , an overall substitution effect of only $25 \%$. While this is a simple illustration, it does capture the essence of the reason for the higher magnitude on the mobile phone use constant in the endogenous ICT use-random coefficient models.

The impact of home computer use on shopping episodes includes both substitution and complementary effects because of the random heterogeneity in responsiveness to home computer use in the random coefficient structure (the standard deviation parameter characterizing this random heterogeneity is significant at the 5\% level). The estimated mean and standard deviation parameters indicate that, among computer users, $74 \%$ make fewer shopping trips than non-users and 26\% make more shopping trips than non-users.

The estimated mean magnitudes of the effects of ICT use on the intershopping hazard in the endogenous ICT use-random coefficients model are provided in the final column of Table 4, and reflect the discussion indicated earlier. Specifically, the exogenous ICT use-random coefficients model underestimate the substitution effect of mobile telephone use considerably. In fact, the results of the endogenous ICT use-random coefficients model indicates a substitution effect for all sociodemographic groups, including the highly educated Karlsruhe residents. 


\subsubsection{Sample selection and overall unobserved heterogeneity results}

The standard deviation of the error term generating sample selection in mobile phone use and the intershopping hazard is highly significant in the endogenous ICT use-random coefficient model. Ignoring this error term leads to a bias in the estimated effect of mobile phone use, as discussed in the previous section. However, the common error term between computer use and the intershopping hazard was not significant and is not included in the table.

The variances of the unobserved heterogeneity terms provide important information regarding the fraction of variation in the intershopping hazard explained by covariates and by unobserved factors. To see this, consider Equation (2) in the context of the general endogenous ICT use-random coefficients specification for the duration hazard:

$$
\begin{aligned}
\lambda_{q i}(\tau) & =\lambda_{0}(\tau) \exp \left(-\alpha_{q}-\beta_{q}^{\prime} x_{q}+\zeta_{q}+\xi_{q}+\Phi_{q i}\right) \\
& =\lambda_{0}(\tau) \exp \left(-\delta^{\prime} w_{q}-v_{q}-\Sigma_{l}\left[\vartheta_{l}+\gamma_{l}^{\prime} y_{q l}+\eta_{q l}\right] x_{q l}+\zeta_{q}+\xi_{q}\right) c_{q i}, \text { where } c_{q i}=\exp \left(\varpi_{q i}\right) \\
& =\lambda_{0}(\tau) \exp \left(-\Pi^{\prime} \tilde{x}_{q}-\sum_{l} \eta_{q l} x_{q l}-v_{q}+\zeta_{q}+\xi_{q}\right) c_{q i},
\end{aligned}
$$

where $\tilde{x}_{q}=\left(w_{q}^{\prime}, x_{q l}, \ldots, x_{q L}, y_{q l} x_{q l}, \ldots, y_{q L} x_{q L}\right)^{\prime}$ and $\Pi=\left(\delta^{\prime}, \vartheta_{l}, \ldots, \vartheta_{L}, \gamma_{l}^{\prime}, \ldots \gamma_{L}^{\prime}\right)^{\prime}$.

Taking the logarithm of both sides of the above equation yields the following equation:

$\ln \lambda_{q i}(\tau)=\ln \lambda_{0}(\tau)-\Pi^{\prime} \tilde{x}_{q}-\sum_{l} \eta_{q l} x_{q l}-v_{q}+\zeta_{q}+\xi_{q}+\ln c_{q i}$.

Since the baseline hazard $\lambda_{0}(\tau)$ is the same across all intershopping spells, the variance across spells of the (log) intershopping hazard can be partitioned as follows:

$\operatorname{Var}\left[\ln \lambda_{q i}(\tau)\right]=\operatorname{Var}\left(\Pi^{\prime} \tilde{x}_{q}\right)+\left[\operatorname{Var}\left(\sum_{l} \eta_{q l} x_{q l}\right)+\operatorname{Var}\left(\nu_{q}\right)+\operatorname{Var}\left(\zeta_{q}\right)+\operatorname{Var}\left(\xi_{q}\right)+\operatorname{Var}\left(\ln c_{q i}\right)\right]$,

where $\operatorname{Var}\left(\Pi^{\prime} \tilde{x}_{q}\right)$ represents the variance due to observed heterogeneity and the second term on the right hand side represents the variance due to unobserved heterogeneity.

The variance due to unobserved heterogeneity comprises only the term $\operatorname{Var}\left(\ln c_{q i}\right)$ if ICT use is considered exogenous to intershopping duration and if randomness in ICT use-effects is 
ignored (we will label this as the exogenous deterministic coefficients model). This term captures overall unobserved heterogeneity across intershopping duration spells and across individuals. The variance due to unobserved heterogeneity, however, can be further partitioned into intraindividual heterogeneity and inter-individual heterogeneity in the exogenous and endogenous random-coefficients models. In both these models, the extent of intra-individual unobserved heterogeneity is provided by $\operatorname{Var}\left(\ln c_{q i}\right)$. In the exogenous ICT use-random coefficients model, the inter-individual unobserved heterogeneity comprises the unobserved variation in response to ICT use $\left[\operatorname{Var}\left(\sum_{l} \eta_{q l} x_{q l}\right)\right]$ and intrinsic inter-individual unobserved heterogeneity $\left[\operatorname{Var}\left(\mathrm{v}_{q}\right)\right]$. In the endogenous ICT use-random coefficient model, the intrinsic inter-individual unobserved heterogeneity effect can be further partitioned into unobserved heterogeneity related to mobile phone use propensity $\left[\operatorname{Var}\left(\zeta_{q}\right)\right]$, unobserved heterogeneity related to home computer use propensity $\left[\operatorname{Var}\left(\xi_{q}\right)\right]$, and unobserved heterogeneity unrelated to ICT use propensity $\left[\operatorname{Var}\left(v_{q}\right)\right]$.

The percentage of variation in the departure time hazard explained by each of the different variance components can be computed from the estimates of $\Pi$ and the estimated variances of the many error components. These percentages are presented in Table 5. The percentage of variation captured by observed and unobserved factors is indicated first. Next, within unobserved heterogeneity, the percentage of variation captured by intra- and interindividual heterogeneity is presented. Thus, the number associated with intra-individual heterogeneity in Table 5 indicates the percentage of unobserved heterogeneity captured by intraindividual heterogeneity. Finally, the inter-individual heterogeneity component is partitioned into its various components. Several important observations may be drawn from this Table. First, the percentage of hazard variation captured by observed factors increases from $11 \%$ in the Exogenous ICT use-deterministic coefficients model to $32 \%$ in the two random coefficients 
model. This is not surprising, since the better representation of inter-individual heterogeneity and sample selection factors allows the influence of observed covariates to be captured more accurately. Second, variation in the hazard due to unobserved factors is higher within the spells of the same individual than across individuals. Specifically, the results show that a little more than $70 \%$ of the total unobserved heterogeneity is due to intra-individual unobserved heterogeneity. Third, the results in the endogenous ICT use-random coefficients model indicate that $85 \%$ of the unobserved individual factors that affect the intershopping hazard also affect mobile phone use propensity. That is, there is a large overlap in unobserved individual factors affecting shopping behavior and mobile phone use propensity.

\subsection{Model Fit Statistics}

The log-likelihood value at convergence for the exogenous ICT use-deterministic coefficients model with 52 parameters is -3151.6 (this model is not presented in the previous section, but forms a base model for comparison). The corresponding values for the exogenous ICT userandom coefficients model with 54 parameters and the endogenous ICT use-random coefficients model with 55 parameters are -3142.4 and -3141.41 , respectively. The log-likelihood value for the naive model that assigns sample shares for ICT use (i.e., uses only constants in the ICT use equations) and adopts a single intershopping hazard profile across all individuals is -3218.2. This naive model has 24 parameters. A likelihood ratio test of the models estimated in this paper with the naive model clearly indicates the significant influence of sociodemographic, locational, and individual-specific unobserved factors on ICT-use and intershopping duration (the likelihood ratio test statistic is of the order of -133.0 , which is larger than the chi-squared statistic with 2830 degrees of freedom at any reasonable level of significance. Similarly, a likelihood ratio test 
between the exogenous ICT use-deterministic coefficients model and the exogenous ICT userandom coefficients model indicates the presence of statistically significant unobserved individual heterogeneity in intershopping duration (the likelihood ratio test statistic is 18.4, which is greater than the chi-squared statistic with 2 degrees of freedom at any reasonable level of significance). The difference between the exogenous ICT use-random coefficients and endogenous ICT use-random coefficients models is statistically significant from a data fit standpoint only at the $15 \%$ level of significance. However, the substantive implications regarding the effect of ICT use on intershopping hazard are substantially different between the two models, as has already been discussed. The fundamental difference between these two models is that the exogenous ICT use-random coefficients model does not allow the unobserved individual-specific determinants of the intershopping hazard to be related to ICT use. However, the endogenous ICT use-random coefficients model indicates that almost all the unobserved individual-specific determinants of intershopping hazard affect ICT use (see Table 5).

\section{CONCLUSION}

This paper has examined the use and travel impacts of two forms of Information and Communication Technologies (ICTs). The two forms of ICTs correspond to mobile telephone use and computer use at home. The travel impacts are examined in the specific context of participation in out-of-home non-maintenance shopping activities over an extended period of six weeks. This is achieved by analyzing the duration between successive shopping participations using a hazard-based structure that recognizes the dynamics of intershopping duration; that is, the structure recognizes that the likelihood of participating in shopping activity depends on the length of time elapsed since the previous participation. 
The econometric model structure developed in the paper accommodates the possible endogeneity of ICT use decisions when individuals make intershopping duration choices. Unlike typical sample selection models in which both the sample selection criteria and the variable of interest are associated with the same unit of observation, the proposed structure recognizes that the criteria for sample selection (i.e., ICT use decisions) are individual-specific, while the resulting sample selection affects all intershopping durations of the individual. The structure also recognizes the interval-level nature of intershopping durations; that is, it recognizes that a day is an interval of time, with several individuals having the same intershopping duration. In addition, the structure allows the responsiveness of shopping activity behavior to ICT use to vary across individuals based on observed and unobserved covariates of the individual. Finally, the structure is able to accommodate unobserved heterogeneity due to both inter-individual as well as intraindividual differences in intershopping durations. To our knowledge, this is the first formulation and application of a duration model in econometric literature to address all the above issues in a comprehensive unifying framework. The resulting model is estimated using a maximum simulated likelihood technique.

The duration formulation is applied to examine ICT use and the impact of ICT use on intershopping duration using a continuous six-week travel survey collected in the cities of Halle and Karlsruhe in Germany in the Fall of 1999. Several results from the empirical analysis in the paper are noteworthy. First, sociodemographic and locational characteristics affect ICT use. Further, there is a strong positive correlation in mobile telephone use and home computer use. Second, the effects of ICTs on activity-travel patterns are mediated by individual sociodemographic and locational factors, as well as by unobserved individual characteristics. Specifically, our results show that the impact of mobile telephones on intershopping duration is a 
function of the education level of the individual and the individual's residence location (i.e., whether the individual resides in Halle or Karlsruhe). The impact of home computer use is mediated by unobserved individual factors, with computer use having a substitution effect on the number of shopping trips for about $78 \%$ of computer users and a complementary effect for about $22 \%$ of computer users. These results emphasize the need to recognize systematic and random variations across individuals in the effect of ICT use on activity-travel behavior. Third, unobserved factors that increase the likelihood of using ICTs are also likely to impact activitytravel behavior. Ignoring this sample selection can and, in general, will lead inappropriate evaluations of the effect of ICT use on activity-travel behavior. In the empirical analysis in the paper, the results showed that the substitution between mobile phone use and shopping travel is grossly underestimated if the effect of common unobserved factors affecting mobile phone use and shopping travel is not considered. Overall, the results suggest a beneficial effect of increasing mobile phone and home computer use penetration in reducing the number of individual trips for non-maintenance related shopping activities. Fourth, the baseline hazard profile indicates a generally increasing trend as the intershopping duration increases. This reflects a "snowballing" effect; that is, the longer the time that has elapsed since the previous shopping participation, the more likely it is to engage in shopping activity. However, the baseline hazard profile is not monotonic and smooth. There is a clear spike at 7 days, a generally elevated profile between 12-16 days, and large spikes at 18 days and 21 days. These results suggest a weekly, biweekly, or triweekly rhythm in shopping participation. Fifth, there is substantial intraindividual variation in intershopping duration. In particular, intra-individual variation represents about $50 \%$ of the overall variation in intershopping hazard, and about $70 \%$ of the unobserved variation in intershopping hazard. This clearly points to the need to observe and analyze 
individual activity-travel behavior over a period of multiple days (see the earlier work of Pas and Koppelman (1987) and Pas (1988) that reach a similar conclusion in the context of day-to-day variability in number of trips; Pas and Sunder (1995), Muthyalagari et al. (2001), Bhat et al. (2002), and Huff and Hanson (1990) also reach a similar conclusion in the context of other dimensions characterizing activity-travel patterns of individuals).

The analysis in this paper provides important insights into the factors influencing ICT use and the effect of ICT use on intershopping duration. However, there are several avenues to extend the analysis. First, the consideration of a comprehensive set of ICTs (rather than just home computer use and mobile telephone use) would provide a broader picture of ICT use effects on activity and travel behavior. Second, a more detailed representation of ICT use, obtained using a communication activity diary, would be valuable in better understanding the interactions between ICT use and activity-travel behavior. Third, extending the current analysis to include multiple activity purposes (such as grocery shopping and recreational activities) would allow consideration of the potential joint nature of participation decisions across activity purposes. Fourth, the analysis may be extended to include a study of the impacts of ICT use on other dimensions of activity-travel behavior, such as length of trips, time-of-day of trips, travel mode used, and activity duration of participation episodes. Fifth, capturing the interaction among household members in activity-travel decisions and studying how these interactions are affected by ICT use is another direction for further research. Finally, the current paper focuses on the activity-travel impacts of ICT use from an individual's perspective. Obviously, reductions in individual travel because of teleshopping will be associated with an increase in delivery travel. The estimation of the net travel impacts of ICT use will therefore require a broader systemwide perspective in analysis (Mokhtarian and Salomon, 2002). 


\section{REFERENCES}

Axhausen, K.W., Zimmerman, Z., Schönfelder, S., Rindsfuser, G. and T. Haupt (2002). Observing the rhythms of daily life: a six week travel diary. Transportation, 29, 2, 95124.

Bhat, C.R. (1996). A hazard-based duration model of shopping activity with nonparametric baseline specification and nonparametric control for unobserved heterogeneity. Transportation Research B, 30, 3, 189-207.

Bhat, C.R. (2000). Duration modeling. In D.A. Hensher and K.J. Button (eds), Handbook of Transport Modelling, Elsevier Science, 91-111.

Bhat, C.R. (2001). Quasi-random maximum simulated likelihood estimation of the mixed multinomial logit model. Transportation Research B, 35, 677-693.

Bhat, C.R., Frusti, T., Zhao, H., Schönfelder, S., and K.W. Axhausen (2002). Intershopping duration: an analysis using multiweek data. Forthcoming in Transportation Research.

Boden, R.J. (1999). Flexible working hours, family responsibilities, and female selfemployment: gender differences in self-employment selection. American Journal of Econometrics and Sociology, 58, 71-83.

Chamberlain, G. (1980). Analysis of covariance with qualitative data. Review of Economic Studies, 47, 225-238.

Diggle, P.J., Liang, K.-Y., and S.L. Zeger (1994). Analysis of Longitudinal Data. Clarendon Press, Oxford, U.K.

Droege, P., ed. (1997). Intelligent Environments. Elsevier, Oxford.

Forrester (2000). B2B Insights: eMarketplaces. Forrester Research, Cambridge MA, http://www.forrester.com/

French, D., et al., eds. (1999). Internet Based Learning: An Introduction and Framework for Higher Education and Business. Stylus, sterling. VA.

Golob, T.F. (2000). TravelBehavior.Com: Activity approaches to modeling the effects of information technology on personal travel behavior. Resource paper, $9^{\text {th }}$ International Association for Travel Behavior Research Conference, July 2-7, Gold Coast, Australia.

Gould, J. and T.F. Golob (1997). Shopping without travel or travel without shopping? An investigation of electronic home shopping. Transport Reviews, 17, 355-376.

Graham, S. and S. Marvin (1996). Telecommunications and the City: Electronic Spaces, Urban Places. Routledge, London.

Greene (2000). Econometric Analysis. Prentice Hall, New Jersey.

Hajivassiliou, A., and P.A. Ruud (1994). Classical estimation methods for LDV models using simulation. In R.F. Engle and D.L. McFadden (eds), Handbook of Econometrics, 4, North Holland, Amsterdam, 2383-2441.

Han, A. and J.A. Hausman (1993). Flexible parametric estimation of duration and competing risk models. Journal of Applied Econometrics, 5, 1-28.

Handy, S. and T. Yantis (1997). The impacts of telecommunications technologies on nonwork travel behavior. Research Report SWUTC/97/721927-1F, Southwest Region University Transportation Center, Center for Transportation Research, The University of Texas at Austin, January.

Harvey, A.S. and M.E. Taylor (2000). Activity settings and travel behaviour: a social contact perspective. Transportation, 27, 1, 53-73. 
Henderson, D.K. and P.L. Mokhtarian (1996). Impacts of center-based telecommuting on travel and emissions: analysis of the Puget Sound Demonstration Project. Transportation Research D, 1, 29-45.

Hensher, D.A. (1999). The valuation of travel time savings for urban car drivers: evaluating alternative model specifications. Technical paper, Institute of Transport Studies, University of Sydney.

Hensher, D.A. and F.L. Mannering (1994). Hazard-based duration models and their application to transport analysis. Transport Reviews, 14, 1, 63-82.

Hsiao, C. (1986). Analysis of Panel Data. Cambridge University Press, Cambridge, MA.

Huff, J.O. and S. Hanson (1990). Measurement of Habitual Behavior: Examining Systematic Variability in Repetitive Travel. In P. Jones (ed), Developments in Dynamic and ActivityBased Approaches to Travel Analysis, Gower Publishing Co., Aldershot, England, 229249.

Johnson and Kotz (1970). Distributions in Statistics: Continuous Univariate Distributions. New York, Wiley, Chapter 21.

Jones, P. and M. Clark (1988). The significance and measurement of variability in travel behaviour. Transportation, 15, 65-87.

Kiefer, N.M. (1988). Econometric duration data and hazard functions. Journal of Econometric Literature, 27, 646-679.

Kilpala, H.K., Seneviratne, P.N., and S.M. Pekkarinen (2000). Electronic grocery shopping and its impact on transportation and logistic with special reference to Finland. Presented at $79^{\text {th }}$ Annual Meeting of the Transportation Research Board, January 9-13, Washington DC.

Koenig, B.E., Henderson, D.K., and P.L. Mokhtarian (1996). The travel and emissions impacts of telecommuting for the State of California Telecommuting Pilot Project. Transportation Research C, 4, 13-32.

Koppelman, F., Salomon, I., and K. Proussaloglou (1991). Teleshopping or store shopping? A choice model for forecasting the use of new telecommunications-based services. Environment and Planning B, 18, 473-489.

Lee, L.-F. (1992). On the efficiency of methods of simulated moments and maximum simulated likelihood estimation of discrete response models. Econometric Theory, 8, 518-552.

Lund, J.R., and P.L. Mokhtarian (1994). Telecommuting and residential location: theory and implications for commute travel in the monocentric metropolis. Transportation Research Record, 1463, 10-14.

Manski, C.F. and I. Salomon (1987). The demand for teleshopping: an application of discrete choice models. Regional Science and Urban Economics, 17, 109-121.

Mannering, J.S. and P.L. Mokhtarian (1995). Modeling the choice of telecommuting frequency in California: an exploratory analysis. Technological Forecasting and Social Change, 49, 49-73.

Martens, M.J. and W. Korver (2000). Forecasting the mobility effects of teleservices using a scenario approach. Presented at $79^{\text {th }}$ Annual Meeting of the Transportation Research Board, January 9-13, Washington DC.

Moberg, A. (1993). Satellitkontor: en studie av kommunikationsmönster vid arbete på distans (in Swedish), Thesis No. 406. Linköping Studies in Science and Technology, Department of Computer and Information Science, Linköping University, Sweden. 
Mokhtarian, P.L., and R. Meenakshisundaram (1999). Beyond tele-substitution: disaggregate longitudinal structural equations modeling of communication impacts. Transportation Research C, 7, 33-52.

Mokhtarian, P.L., and I. Salomon (1997). Modeling the desire to telecommute: the importance of attitudinal factors in behavioral models. Transportation Research A, 31, 1, 35-50.

Mokhtarian, P.L., and I. Salomon (2002). Emerging travel patterns: do telecommunications make a difference? In H.S. Mahmassani (ed), In Perpetual Motion: Travel Behavior Research Opportunities and Application Challenged, Pergamon, Elsevier Science, Oxford, U.K., 143-182.

Mokhtarian, P.L., and K.V. Varma (1998). The trade-off between trips and distance traveled in analyzing the emissions of center-based telecommuting. Transportation Research D, 3, 419-428.

Muthyalagari, G.R., Parashar, A., and R.M. Pendyala (2001). Measuring Day-to-Day Variability in Travel Characteristics Using GPS Data. Presented at the $80^{\text {th }}$ Annual Meeting of the Transportation Research Board, Washington D.C., January.

Pas, E.I. (1988). Weekly Travel-Activity Behavior. Transportation, Vol. 15, pp. 89-109.

Pas, E.I. and F.S. Koppelman (1987). An Examination of the Determinants of Day-to-Day Variability in Individuals' Urban Travel Behavior. Transportation, Vol. 14, pp. 3-20.

Pas, E.I. and S. Sundar (1995). Intra-personal Variability in Daily Urban Travel Behavior: Some Additional Evidence. Transportation, Vol. 22, pp. 135-150.

Pendyala, R.M., Goulias, K.G., and R. Kitamura (1991). Impact of telecommuting on spatial and temporal patterns of household travel. Transportation, 18, 383-409.

Pioneer Consulting (1999). Next Generation Broadband Satellite Networks: A Market and Technology Assessment Report. Pioneer Consulting, Cambridge, MA.

Salomon, I. (1996). Telecommunications, cities and technological opportunism. Annals of Regional Science, 30, 75-90.

Schlich, R., König, A., Aschwanden, A., Kaufmann, A., und K.W. Axhausen (2000). Mobidrive: Data format guide, Arbeitsberichte Verkehrs- und Raumplanung, 35, Institut für Verkehrsplanung, Transporttechnik, Strassen- und Eisenbahnbau, ETH Zürich, Zürich.

Spittje, H.D. (1994). Tele/homework and time use/travel behaviour. Paper for presentation at IATAR Conference, July 18-23, Bielefeld, Germany. Contact: H.D.Spittje@ trc.rug.nl.

Tacken, M. (1990). Effects of teleshopping on the use of time and space. Transportation Research Record, 1285, 89-91.

Train, K. (1999). Halton sequences for mixed logit. Technical paper, Department of Economics, University of California, Berkeley.

Viswanathan, K. and K.G. Goulias (2001). Travel behavior implications of information and communications technologies (ICT) in the Puget Sound region. Presented at $80^{\text {th }}$ Annual Meeting of the Transportation Research Board, January 7-11, Washington DC.

Yen, J-R and H.S. Mahmassani (1998). Telecommuting adoption: conceptual framework and model estimation. Transportation Research Record 1606, 95-102.

Yim, Y. (2000). Telecommunications and travel behavior: would cellular communication generate more trips? Presented at $79^{\text {th }}$ Annual Meeting of the Transportation Research Board, January 9-13, Washington DC. 
Zimmerman, C.A., Campbell, J.L., and C. Cluett (2001). Impact of new information and communication technologies on transportation agencies: a synthesis of highway practice. NCHRP Synthesis 296, National Cooperative Highway Research Program, Transportation Research Board.

Zumkeller, D. (1996). Communication as an element of the overall transport context - an empirical study. In A.J. Richardson, E.S. Ampt and A.H. Meyburg (eds), Proceedings of the Fourth International Conference on Survey Methods in Transport, 66-83, Eucalyptus Press, Melbourne. 


\section{LIST OF FIGURES}

Figure 1. Sample and Baseline Hazard Functions

\section{LIST OF TABLES}

Table 1. Variable Definitions and Sample Statistics (Number of individuals $=255$ )

Table 2. Mobile Phone Use and Computer Use Propensity

Table 3. Hazard Duration Model Results

Table 4. Effect of ICT Use on Intershopping Hazard

Table 5. Percentage of Intershopping Hazard Variation Explained by Observed and Unobserved Factors 


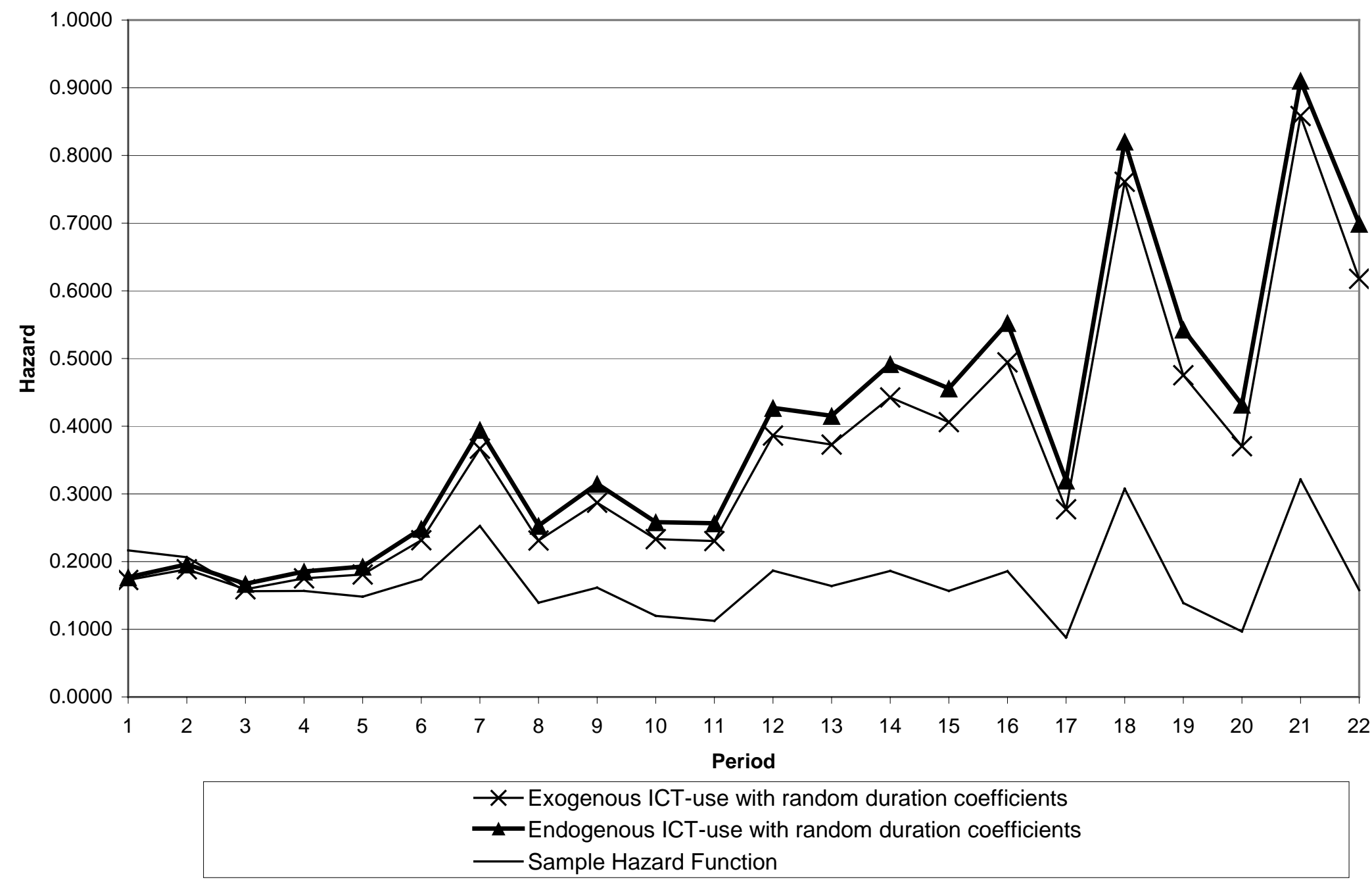

Figure 1. Sample and Baseline Hazard Functions 
Table 1. Variable Definitions and Sample Statistics (Number of individuals $=\mathbf{2 5 5}$ )

\begin{tabular}{|c|c|c|c|}
\hline Variable & Definition & Mean & $\begin{array}{l}\text { Std. } \\
\text { Dev. }\end{array}$ \\
\hline \multicolumn{4}{|l|}{ Individual characteristics } \\
\hline Employed & 1 if individual is employed, 0 otherwise & 0.60 & 0.49 \\
\hline High education & 1 if individual has attended technical college or university, 0 otherwise & 0.30 & 0.46 \\
\hline Age $16-20$ years & 1 if individual is between 16 and 20 years of age, 0 otherwise & 0.09 & 0.29 \\
\hline Age 20-29 years & 1 if individual is between 20 and 29 years of age, 0 otherwise & 0.14 & 0.34 \\
\hline Age $30-49$ years & 1 if individual is between 30 and 49 years of age, 0 otherwise & 0.40 & 0.49 \\
\hline Age $>50$ years & 1 if individual is over 50 years of age, 0 otherwise & 0.37 & 0.48 \\
\hline Male & 1 if individual is male, 0 otherwise & 0.48 & 0.50 \\
\hline Primary mode used is car & 1 if car is the most frequently used mode for shopping, 0 otherwise & 0.60 & 0.49 \\
\hline Fraction of shopping episodes chained & $\begin{array}{l}\text { Fraction of total non-maintenance shopping episodes that are chained } \\
\text { with other activities }\end{array}$ & 0.51 & 0.35 \\
\hline \multicolumn{4}{|l|}{ Household characteristics } \\
\hline Income (in thousands of DM) & Monthly household income & 4.32 & 2.05 \\
\hline Number of adults & Number of adults in household & 2.26 & 0.83 \\
\hline Nuclear family & 1 if household is a nuclear family, 0 otherwise & 0.44 & 0.50 \\
\hline Couple family & 1 if household is a couple family, 0 otherwise & 0.32 & 0.47 \\
\hline \multicolumn{4}{|l|}{ Residential location characteristics } \\
\hline Karlsruhe & 1 if home is in Karlsruhe, 0 if home is in Halle & 0.58 & 0.49 \\
\hline Urban-suburban residence & 1 if home is in an urban-suburban area, 0 otherwise & 0.42 & 0.49 \\
\hline Suburban residence & 1 if home is in a suburban area, 0 otherwise & 0.23 & 0.42 \\
\hline \multicolumn{4}{|l|}{ ICT use } \\
\hline Mobile phone use & 1 if individual has a mobile phone available for use, 0 otherwise & 0.36 & 0.48 \\
\hline Home computer use & 1 if individual has a private email address, 0 otherwise & 0.22 & 0.41 \\
\hline
\end{tabular}


Table 2. Mobile Phone Use and Computer Use Propensity

\begin{tabular}{|c|c|c|c|c|}
\hline \multirow[t]{2}{*}{ Variable } & \multicolumn{2}{|c|}{$\begin{array}{l}\text { Exogenous ICT-use with } \\
\text { random coefficients hazard }\end{array}$} & \multicolumn{2}{|c|}{$\begin{array}{l}\text { Endogenous ICT-use with } \\
\text { random coefficients hazard }\end{array}$} \\
\hline & Parameter & t-statistic & Parameter & t-statistic \\
\hline \multicolumn{5}{|l|}{ Mobile Phone Use Propensity } \\
\hline Constant & -0.2279 & -0.438 & -0.2796 & -0.510 \\
\hline $\begin{array}{l}\text { Individual characteristics } \\
\text { Employed } \\
\text { High education } \\
\text { Age (Base is } 16-19 \text { years) } \\
\text { 20-29 years } \\
\text { 30-49 years } \\
\text { > 50 years }\end{array}$ & $\begin{array}{l}0.5236 \\
0.5118 \\
-1.3804 \\
-0.5510 \\
-0.8655 \\
\end{array}$ & $\begin{array}{r}2.680 \\
2.177 \\
-2.944 \\
-1.476 \\
-2.326 \\
\end{array}$ & $\begin{array}{l}0.6210 \\
0.5294 \\
-1.4574 \\
-0.6326 \\
-0.9603 \\
\end{array}$ & $\begin{array}{r}2.873 \\
2.098 \\
-2.972 \\
-1.605 \\
-2.448 \\
\end{array}$ \\
\hline $\begin{array}{l}\text { Household characteristics } \\
\text { Income } \\
\text { Number of adults } \\
\text { Nuclear family }\end{array}$ & $\begin{array}{r}0.0694 \\
-0.2785 \\
1.2175 \\
\end{array}$ & $\begin{array}{r}1.359 \\
-1.853 \\
4.186 \\
\end{array}$ & $\begin{array}{r}0.0760 \\
-0.3030 \\
1.3181 \\
\end{array}$ & $\begin{array}{r}1.405 \\
-1.875 \\
4.169 \\
\end{array}$ \\
\hline $\begin{array}{l}\text { Residential location characteristics } \\
\text { Karlsruhe } \\
\text { Urban-suburban residence } \\
\text { Suburban residence }\end{array}$ & $\begin{array}{l}0.2700 \\
0.4484 \\
0.4030\end{array}$ & $\begin{array}{l}1.280 \\
1.948 \\
1.702 \\
\end{array}$ & $\begin{array}{l}0.2970 \\
0.5080 \\
0.4583\end{array}$ & $\begin{array}{l}1.318 \\
2.081 \\
1.789\end{array}$ \\
\hline \multicolumn{5}{|l|}{ Computer Use Propensity } \\
\hline Constant & 0.2868 & 0.528 & 0.2931 & 0.540 \\
\hline $\begin{array}{l}\text { Individual characteristics } \\
\text { High education }\end{array}$ & 0.6829 & 2.671 & 0.6853 & 2.686 \\
\hline $\begin{array}{l}\text { Household characteristics } \\
\text { Income } \\
\text { Number of adults } \\
\text { Nuclear family } \\
\text { Single person household }\end{array}$ & $\begin{array}{r}0.1236 \\
-0.8486 \\
0.7084 \\
-0.8428 \\
\end{array}$ & $\begin{array}{r}2.385 \\
-3.708 \\
2.071 \\
-2.200 \\
\end{array}$ & $\begin{array}{r}0.1238 \\
-0.8523 \\
0.7143 \\
-0.8423 \\
\end{array}$ & $\begin{array}{r}2.387 \\
-3.722 \\
2.088 \\
-2.201 \\
\end{array}$ \\
\hline $\begin{array}{l}\text { Residential location characteristics } \\
\text { Karlsruhe }\end{array}$ & 0.5583 & 2.707 & 0.5580 & 2.709 \\
\hline $\begin{array}{l}\text { Unobserved correlation between mobile } \\
\text { phone and computer-use propensities }\end{array}$ & 0.3395 & 2.588 & 0.3569 & 2.480 \\
\hline
\end{tabular}


Table 3. Hazard Duration Model Results

\begin{tabular}{|c|c|c|c|c|}
\hline \multirow[t]{2}{*}{ Variable } & \multicolumn{2}{|c|}{$\begin{array}{l}\text { Exogenous ICT use with } \\
\text { random coefficients hazard }\end{array}$} & \multicolumn{2}{|c|}{$\begin{array}{l}\text { Endogenous ICT use with } \\
\text { random coefficients hazard }\end{array}$} \\
\hline & Parameter & t-statistic & Parameter & t-statistic \\
\hline $\begin{array}{l}\text { Individual characteristics } \\
\text { Male }\end{array}$ & 0.2400 & 2.031 & 0.2385 & 1.974 \\
\hline Primary mode used is car & 0.2764 & 2.339 & 0.2752 & 2.358 \\
\hline$\%$ of shopping episodes chained & -0.4264 & -2.550 & -0.4670 & -2.638 \\
\hline $\begin{array}{l}\text { Household characteristics } \\
\text { Income }\end{array}$ & -0.0250 & -0.851 & -0.0335 & -1.091 \\
\hline Couple family & -0.2428 & -2.114 & -0.2155 & -1.802 \\
\hline \multicolumn{5}{|l|}{$\begin{array}{l}\text { ICT use variables } \\
\text { Mobile phone use }\end{array}$} \\
\hline Constant & 0.3898 & 2.037 & 0.6321 & 2.855 \\
\hline High education & -0.2714 & -1.296 & -0.2606 & -1.213 \\
\hline Karlsruhe & -0.3002 & -1.382 & -0.3289 & -1.438 \\
\hline \multicolumn{5}{|l|}{ Computer use } \\
\hline Constant & 0.2577 & 1.492 & 0.2465 & 1.297 \\
\hline Standard deviation & 0.3358 & 1.507 & 0.3898 & 1.670 \\
\hline $\begin{array}{l}\text { Sample selection and heterogeneity terms } \\
\text { Common unobserved error component between mobile } \\
\text { phone use propensity and duration hazard }\end{array}$ & - & - & 0.4174 & 4.468 \\
\hline \multicolumn{5}{|l|}{ Unobserved heterogeneity } \\
\hline Across individuals & 0.4040 & 6.394 & 0.0900 & 0.561 \\
\hline Across spells & 0.6224 & 2.765 & 0.6602 & 3.037 \\
\hline
\end{tabular}


Table 4. Effect of ICT Use on Intershopping Hazard

\begin{tabular}{|l||c|c||}
\hline \multicolumn{1}{|c||}{ ICT use } & \multicolumn{2}{c|}{ Mean percentage change in hazard in... } \\
\cline { 2 - 3 } & $\begin{array}{c}\text { Exogenous ICT use with } \\
\text { random coefficients }\end{array}$ & $\begin{array}{c}\text { Endogenous ICT use with } \\
\text { random coefficients }\end{array}$ \\
\hline \hline $\begin{array}{l}\text { Mobile telephone use } \\
\text { Low education, Halle residence }\end{array}$ & -32 & -47 \\
High education, Halle residence & -11 & -31 \\
Low education, Karlsruhe residence & -8 & -26 \\
High education, Karlsruhe residence & +20 & -4 \\
Computer use & -23 & -19 \\
\hline \hline
\end{tabular}


Table 5. Percentage of Intershopping Hazard Variation Explained by Observed and Unobserved Factors

\begin{tabular}{|c|c|c|}
\hline \multirow{2}{*}{ Heterogeneity source } & \multicolumn{2}{|c|}{$\begin{array}{c}\text { Percentage of total intershopping hazard variation } \\
\text { explained by each heterogeneity source in... }\end{array}$} \\
\hline & $\begin{array}{l}\text { Exogenous ICT use with } \\
\text { random coefficients model }\end{array}$ & $\begin{array}{l}\text { Endogenous ICT use with } \\
\text { random coefficients model }\end{array}$ \\
\hline Observed heterogeneity & 32 & 32 \\
\hline Unobserved heterogeneity & 68 & 68 \\
\hline Intra-individual & 73 & 72 \\
\hline Inter-individual & 27 & 28 \\
\hline$\underline{\text { Response to home computer use }}$ & $\underline{9}$ & $\underline{11}$ \\
\hline Intrinsic heterogeneity related to mobile phone use & - & $\underline{85}$ \\
\hline Intrinsic heterogeneity unrelated to ICT use & $\underline{91}$ & $\underline{4}$ \\
\hline
\end{tabular}

